\title{
Endoscopic Cholangiopancreatography Related Perforation with Tell-Tale Radiological Signs
}

\author{
Saket Kumar ${ }^{1 *}$, Ritu Singh ${ }^{2}$ and Abhijit Chandra ${ }^{1}$ \\ ${ }^{1}$ Department of Surgical Gastroenterology, KGMU, Lucknow, India \\ ${ }^{2}$ Department of Critical Care Medicine, SGPGIMS, Lucknow, India
}

Received: April 04, 2018; Published: May 11, 2018

*Corresponding author: Saket Kumar, Department of Surgical Gastroenterology, King George’s Medical University, India; Tel: +919621502507;

Fax: +91-522-2256116; Email: krsaketsingh@gmail.com

\begin{abstract}
Endoscopic retrograde cholangiopancreatogram (ERCP) related duodenal perforations are uncommon but serious complication. Early diagnosis and management are crucial in decreasing the morbidity and mortality of iatrogenic duodenal perforations. Plain abdominal X-ray in conjunction with bedside clinical signs may help in making a rapid diagnosis. The management depends on the type of perforation and ranges from conservative treatment to emergency laparotomy. Here, we discuss a case of an iatrogenic duodenal perforation (Stapfer type II) that was diagnosed with Plain abdominal X-ray showing typical signs of retroperitoneal perforation. The patient was managed conservatively with nonsurgical means.
\end{abstract}

Keywords: Endoscopic retrograde cholangiopancreatography; Iatrogenic; Duodenal perforation; Pneumoretroperitoneum

Abbreviations: ERCP: Endoscopic Retrograde Cholangiopancreatography

\section{Introduction}

Endoscopic Retrograde Cholangiopancreatography (ERCP) has become a routine procedure for diagnosis and management of pancreatico-biliary diseases. Though considered a safe procedure, it has its own set of risk and potential complications. ERCP related perforation, a potentially life-threatening complication, necessitates timely diagnosis and treatment [1]. This present case illustrates the importance of plain abdominal $\mathrm{X}$-ray in diagnosing an ERCP-related perforation and guiding early management.

\section{Case Report}

A 49-year-old female presented with 2 months history of right hypochondrial pain, progressive jaundice and pruritus. Computed tomography scan revealed gallbladder neck tumour infiltrating the adjacent liver parenchyma and common hepatic duct with few metastatic omental deposits. The patient was admitted and planned for palliative biliary stenting. Cholangiography done after biliary cannulation revealed a mid CBD stricture. Guide wire was manoeuvred above stricture with difficulty, but stent could not be passed through the stricture. During procedure, patient developed severe abdominal pain and distension. Abdominal skiagram done shortly after the procedure revealed tell-tale signs of retroperitoneal duodenal perforation. The presence of retroperitoneal air (more on the right side), reniform pattern outlining kidneys, delineation of bilateral psoas muscle borders, triangle sign, and bowel showing double wall sign (Rigler's sign) were characteristic of ERCP related perforation.

Thin rim of air could also be seen under the right dome of diaphragm (Figure 1 \& 2). Patient was diagnosed with retroperitoneal duodenal perforation and was initiated conservative management in form of antibiotics, intravenous fluids and nasogastric aspiration. However, she developed signs of sepsis (fever, elevated leucocyte counts) on day 2. An abdominal sonogram revealed moderate amount of retroperitoneal fluid collection. A pigtail catheter was placed in the retroperitoneum to drain the collection. Patient responded favourably to drainage and her fever subsided. She was discharged from hospital on day 9 in stable condition. She underwent palliative percutaneous transhepatic biliary drainage at a later date. She survived for 5 months after which she succumbed to advanced malignancy. 


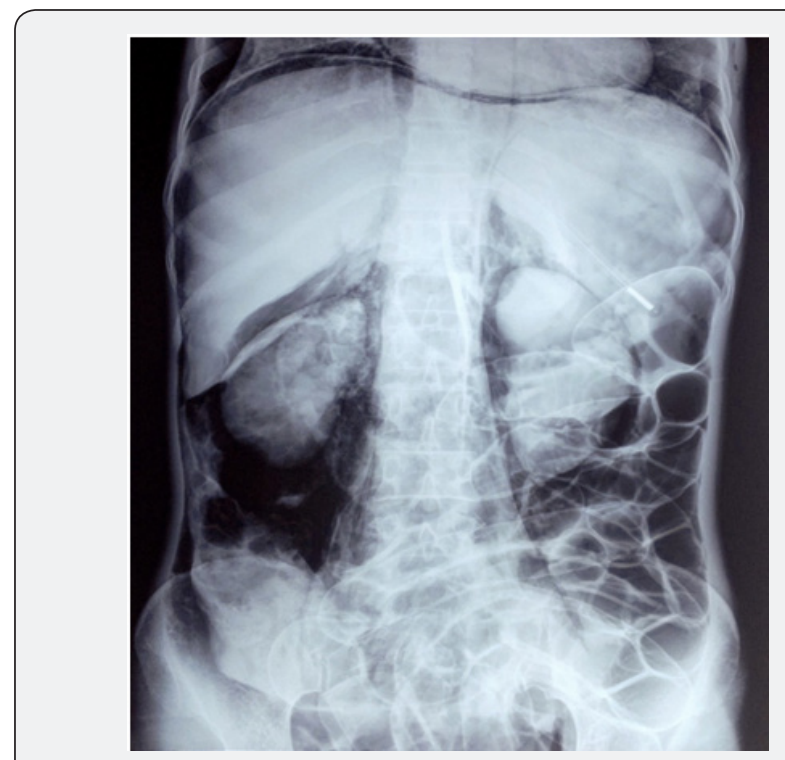

Figure 1: Plain X-ray abdomen of patient showing signs of ERCP related perforation.

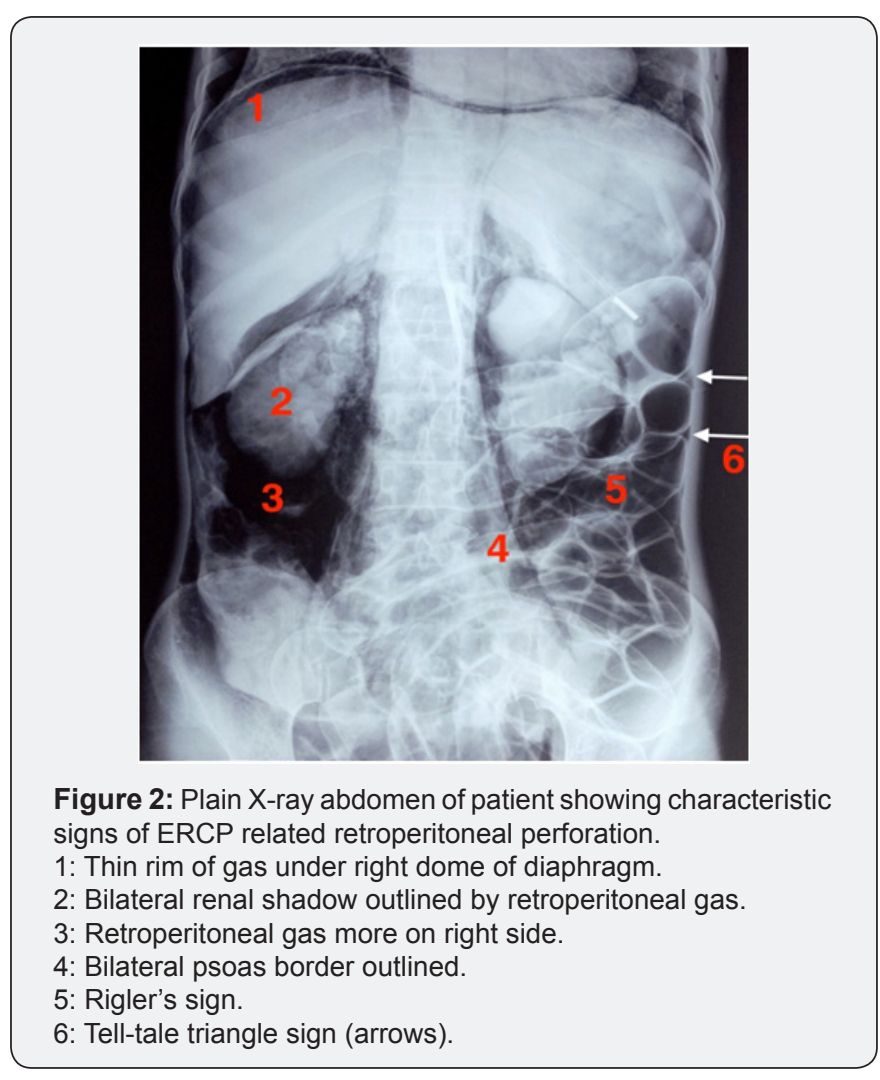

\section{Discussion}

ERCP is considered the standard procedure for the diagnosis and treatment of pancreatico-biliary pathologies. It is commonly used for preoperative biliary drainage and palliation of jaundice in bilio-pancreatic malignancies. ERCP is widely regarded as a safe procedure, but the associated rate of complications such as post-ERCP pancreatitis, hemorrhage, cholangitis and perforation approaches $4 \%-16 \%$ [2-6]. Perforation is a rare but potentially life-threatening complication. Recent studies have reported the incidence of duodenal perforations between $0.8 \%$ and $1.5 \%$ and mortality rate up to $1.5 \%$ [3-6]. The diagnosis of duodenal perforation is usually made during the procedure.

However, if there is doubt about perforation at ERCP, an immediate contrast upper gastrointestinal study or computed tomography scan should be obtained [7]. Free or retroperitoneal air alone can be detected even in plain skiagram. Abdominal $\mathrm{X}$-ray is a quick and inexpensive procedure that can be done bedside as well. Stapfer et al. classified ERCP-related perforations into four types based on mechanism, anatomical location, and severity of the injury. The preferred treatment modality also depends on the type of the perforation [8]. Type I duodenal injuries are perforations of the lateral or medial wall, caused by the endoscope itself. These are usually large perforations and require urgent surgery.

Type II duodenal injuries are peri-vaterian or periampullary perforations, and mostly occur during endoscopic sphincterotomy. Majority of these injuries can be managed conservatively and surgery is required in selected cases with large leaks. Type III duodenal injuries are perforations of the distal bile duct, typically due to guide wire manipulation. These are small perforations and do not require surgical intervention.

Type IV duodenal injuries are minute retroperitoneal perforations characterized by retroperitoneal air only. These are not true perforation and occur due to excess use of compressed air. These are fairly common and usually asymptomatic, and have been reported in up to $40 \%$ of cases [9]. Presence of air in retro peritoneum is not predictive of surgery and most of these cases can be managed conservatively [5-8]. The majority of ERCPrelated periampullary or biliary perforations can be managed non-surgically [7]. Retropneumoperitoneum alone occurs frequently after ERCP and should not raise alarm in absence of sepsis, significant abdominal collection or peritonitis.

Image guided drainage of collection is indicated in patients with increasing abdominal pain, sepsis or failure to improve after initial medical management. The indication for surgery is limited to perforations with a major contrast medium leak, severe sepsis despite nonsurgical management and peritoneal collections that cannot be tackled by nonsurgical means $[7,8]$. To conclude, an abdominal X-ray may prove to be a useful tool for timely diagnosis of ERCP-related perforation and guiding its management, if the treating surgeon is familiar with the characteristic skiagram signs.

\section{References}

1. CD Jefferiss (1972) Spontaneous rupture of the stomach in an adult. $\mathrm{Br}$ J Surg 59(1): 79-80.

2. Adson DE, Mitchell JE, Trenkner SW (1997) The superior mesenteric artery syndrome and acute gastric dilatation in eating disorders: a case report of two cases and review of the literature. Int J Eat Disord 21(2): 103-114. 
3. Abdu RA, Garritano D, Culver O (1987) Acute gastric necrosis in anorexia nervosa and bulimia. Two case reports. Arch Surg 122(7): 830-832.

4. Todd SR, Marshall GT, Tyroch AH (2000) Acute gastric dilatation revisited. Am Surg 66(8): 709-710.
5. Somerwell TH (1945) Physiologic gastrectomy. Br J Surg 33: 146.

6. Byrne JJ, Cahill JM (1961) Acute gastric dilatation. The American Journal of Surgery 101(3): 301-309.

\section{Your next submission with Juniper Publishers will reach you the below assets}

- Quality Editorial service

- Swift Peer Review

- Reprints availability

- E-prints Service

- Manuscript Podcast for convenient understanding

- Global attainment for your research

- Manuscript accessibility in different formats

( Pdf, E-pub, Full Text, Audio)

- Unceasing customer service

Track the below URL for one-step submission https://juniperpublishers.com/online-submission.php 